\title{
Exclusive breakup of ${ }^{17} \mathrm{~F}$ on ${ }^{58} \mathrm{Ni}$ and ${ }^{208} \mathrm{~Pb}$ within the continuum-discretized coupled-channels method
}

\author{
Y. Kucuk ${ }^{*}$ \\ Department of Physics, University of Surrey, Guildford, Surrey GU2 7XH, United Kingdom, and Department of Physics, \\ Giresun University, Giresun, Turkey
}

A. M. Moro ${ }^{\dagger}$

Departamento de FAMN, Facultad de Física, Universidad de Sevilla, Apartado 1065, E-41080 Sevilla, Spain

(Received 21 November 2011; revised manuscript received 19 July 2012; published 4 September 2012)

\begin{abstract}
An analysis of the elastic and exclusive breakup of ${ }^{17} \mathrm{~F}$ on ${ }^{58} \mathrm{Ni}$ and ${ }^{208} \mathrm{~Pb}$ targets is presented. The study is undertaken using the continuum-discretized coupled-channels (CDCC) method, assuming a core + valence model for the ${ }^{17} \mathrm{~F}$ projectile. The results are compared with recent data published for these reactions [Phys. Lett. B 681, 22 (2009)]. The calculations show that the exclusive breakup cross sections contain contributions from high multipoles, as well as a complicated interplay between Coulomb and nuclear couplings. This makes it difficult to extract radiative capture cross sections or structure information from the analyzed data.
\end{abstract}

DOI: 10.1103/PhysRevC.86.034601

PACS number(s): 24.10.Eq, 25.60.Gc, 25.70.Bc

\section{INTRODUCTION}

Coulomb breakup experiments, in which a projectile nucleus is dissociated into its constituents when colliding with a heavy target, provide a useful tool for studying capture reactions in situations in which the direct measurement is difficult or impossible. This technique relies on the fact that the breakup cross section of a projectile is proportional to the cross section for photodissociation [1], and hence the radiative capture cross sections can be obtained via detailed balance. A difficulty inherent in this approach is the fact that the capture cross section is typically largely dominated by the $E 1$ Coulomb multipole, whereas the breakup experiment may contain contributions from higher Coulomb multipoles (e.g., E2, E3), as well as from nuclear breakup. These effects complicate the connection between both processes and hence the usefulness of the method to extract reliable capture cross sections. A key example for which this technique has been applied is provided by the capture reaction ${ }^{7} \mathrm{Be}(p, \gamma)[2-4]$, which is considered to be the most important for the standard solar model.

Coulomb dissociation experiments have been analyzed using a variety of approaches. At sufficiently high energies and forward angles, perturbative calculations, such as those based on the semiclassical theory of Alder and Winther of Coulomb excitation [5], have been used $[2,4,6]$. Nonperturbative semiclassical methods have been also developed. Some of these methods perform a numerical integration of the time-dependent (TD) Schrödinger equation [7-9], assuming that the projectile-target motion follows a classical trajectory, while the projectile dynamics is treated quantum mechanically to all orders. This method allows evaluation of both Coulomb and nuclear contributions to breakup in a unitary manner. More recently, Bonaccorso and collaborators have proposed a semiclassical model which treats both Coulomb

\footnotetext{
*y.kucuk@surrey.ac.uk

†moro@us.es
}

and nuclear to all orders but assumes a straight-line trajectory for the motion of the composite projectile. The method has been applied to both neutron [10] and proton-halo two-body projectiles [11].

Another approach successfully used to interpret Coulomb dissociation experiments $[12,13]$ is the continuum-discretized coupled-channels (CDCC) method [14]. This method is fully quantal and does not make any classical approximation for the projectile-target motion. Moreover, it takes into account both Coulomb and nuclear breakup and higher order effects. A comparison between the CDCC method and several nonperturbative semiclassical approaches has been recently presented in Ref. [15]. For angle-integrated observables, the authors found good agreement between the CDCC method and the TD method of Ref. [8] at energies as low as $20 \mathrm{MeV} /$ nucleon. However, for angular distributions significant differences were found, which were attributed to the use of classical trajectories in the TD method. The comparison was nevertheless restricted to neutron-halo nuclei and hence the conclusions cannot be readily extended to proton-halo nuclei, for which the Coulomb breakup mechanism is very different.

A recent application of the TD method to Coulomb dissociation of a proton-valence nucleus is provided in the work of Liang et al. [16], who measured the dissociation of the ${ }^{17} \mathrm{~F}$ nucleus on ${ }^{208} \mathrm{~Pb}$ and ${ }^{58} \mathrm{Ni}$ targets. The extracted exclusive breakup angular distributions were compared with two types of calculations: a first-order perturbation calculation for Coulomb dissociation, including $E 1$ and $E 2$ transitions, and a TD dynamical calculation similar to that of Ref. [8]. The authors found that the $E 1+E 2$ first-order calculation for Coulomb dissociation reproduces reasonably well the ${ }^{58} \mathrm{Ni}$ data, but it significantly overpredicts the ${ }^{208} \mathrm{~Pb}$ data. Qualitatively similar conclusions are found for the TD calculations; that is, they reproduce reasonably well the ${ }^{58} \mathrm{Ni}$ data, but they tend to overestimate the ${ }^{208} \mathrm{~Pb}$ data by a factor of 2 . For this target, the authors present an additional perturbation calculation, in which the proton separation energy of ${ }^{17} \mathrm{~F}$ is arbitrarily increased to 1.2 $\mathrm{MeV}$, following a suggestion in a previous work [17]. 
With this effective binding energy, the ${ }^{208} \mathrm{~Pb}$ data are well reproduced.

In this work, we present an analysis of the data of Ref. [16] in terms of the continuum-discretized coupledchannels (CDCC) method [14]. The goal of these calculations is to assess the relevance of higher order effects, the relative importance of nuclear and Coulomb breakup, and the contribution of different multipoles on the breakup cross sections. From this study, we aim to examine the potential usefulness of this reaction as a tool to extract indirect information on the radiative capture cross sections.

Taking advantage of the fact that the CDCC method treats exactly the Coulomb part of the proton interaction with the core and the target, we investigate also the role of the proton-target Coulomb potential as well as the accuracy of the approximation of treating the valence proton as a neutron with an effective separation energy, according to the suggestion of Ref. [17].

The paper is structured as follows. In Sec. II we introduce the three-body model used in the CDCC calculations. In Sec. III we compare these calculations with the quasielastic and exclusive breakup data from Ref. [16]. In Sec. IV we discuss the proton and core angular distributions obtained from the CDCC calculations. In Sec. V we investigate the contribution of different multipoles to the calculated breakup cross sections. In Sec. VI we analyze the relative importance of Coulomb and nuclear breakup as well as the interplay of the nuclear and Coulomb parts of the proton-target interaction. Finally, Sec. VII is devoted to summary and conclusions.

\section{THE EFFECTIVE HAMILTONIAN}

\section{A. Structure model for ${ }^{17} \mathbf{F}$}

The two reactions considered here, ${ }^{17} \mathrm{~F}+{ }^{58} \mathrm{Ni}$ and ${ }^{17} \mathrm{~F}+$ ${ }^{208} \mathrm{~Pb}$, are treated within a three-body model $\left(p+{ }^{16} \mathrm{O}+{ }^{58} \mathrm{Ni}\right.$ and $\left.p+{ }^{16} \mathrm{O}+{ }^{208} \mathrm{~Pb}\right)$, which is based on a simple valence + core picture of the ${ }^{17} \mathrm{~F}$ projectile. The ${ }^{17} \mathrm{~F}$ states are treated as single-particle configurations of the valence proton coupled to the ${ }^{16} \mathrm{O}$ ground state. Thus, the ${ }^{17} \mathrm{~F}$ ground state $\left(5 / 2^{+}\right)$and the bound excited state $\left(1 / 2^{+}\right)$are described in terms of pure $1 d_{5 / 2}$ and $2 s_{1 / 2}$ configurations, respectively. The ${ }^{17} \mathrm{~F}$ wave functions are taken as the eigenstates of a WoodsSaxon potential (with central and spin-orbit components) with parameters taken from Ref. [18]. This potential reproduces the separation energy of the ground state and the bound excited state $\left(E_{x}=0.495 \mathrm{MeV}\right)$ as well as the position of the $3 / 2^{+}$resonance at $E_{x}=5 \mathrm{MeV}$. Continuum states with orbital angular momentum $\ell$ were also included and their eigenfunctions were generated with the same $p+{ }^{16} \mathrm{O}$ potential used for the bound states. For each value of $\ell$, the continuum was truncated at a maximum excitation energy and discretized into energy bins, following the standard average method used in CDCC calculations. As a result of this procedure, the projectile spectrum is represented by a finite set of states that we denote generically as $\left\{\phi_{\alpha}(\mathbf{r})\right\}$, which includes the two bound states and the discrete representation of the continuum (the index $\alpha$ comprises all the quantum numbers required to specify the state). The maximum excitation energy and the number of bins were increased progressively until convergence of the calculated observables was achieved.

\section{B. The proton-target and ${ }^{16} \mathrm{O}$-target interactions}

For the CDCC calculations one needs to specify also the proton-target and ${ }^{16} \mathrm{O}$-target interactions, which are used to generate the so-called coupling potentials, given by

$$
V_{\alpha ; \alpha^{\prime}}=\left\langle\phi_{\alpha}(\mathbf{r})\left|V_{c t}\left(\mathbf{R}_{c t}\right)+V_{p t}\left(\mathbf{R}_{p t}\right)\right| \phi_{\alpha^{\prime}}(\mathbf{r})\right\rangle,
$$

where $V_{c t}\left(\mathbf{R}_{c t}\right)$ and $V_{p t}\left(\mathbf{R}_{p t}\right)$ are the total (nuclear and Coulomb) interactions between the core-target and protontarget systems, respectively. These interactions are typically represented by optical potentials describing the elastic scattering at the appropriate energy per nucleon. It is worth recalling that, with this choice of the fragment-target interactions, the corresponding CDCC three-body wave function contains only configurations in which both the valence and the core are elastically scattered by the target. This corresponds to the so-called elastic breakup. Processes in which any of the two fragments is either absorbed or excited during the collision are excluded from the CDCC model space. However, the influence of these nonelastic breakup channels (sometimes denoted stripping) on the calculated observables is effectively taken into account by the imaginary parts of the underlying optical potentials. The experimental angular distributions used in this work correspond to exclusive measurements in which nonelastic breakups are excluded, so the comparison with the CDCC method is justified.

In order to study the sensitivity of the calculated observables with respect to these fragment-target interactions, two different sets of potentials were considered. For the proton- ${ }^{58} \mathrm{Ni}$ and proton- ${ }^{208} \mathrm{~Pb}$ systems we used the global parametrizations of Koning-Delaroche (denoted $P 1$ hereafter) [19] and BechettiGreenless (denoted P2) [20]. For both parametrizations, the Coulomb potential was taken as that of a uniformly charged sphere with reduced radius $r_{c}=1.2 \mathrm{fm}$. For simplicity, the spin-orbit part of these interactions is not included. The parameters for the $P 1$ and $P 2$ potentials, evaluated at $E_{p}=$ $10 \mathrm{MeV}$, are given in Table I.

For the ${ }^{16} \mathrm{O}+$ target interaction we have also used two different parametrizations, denoted $C 1$ and $C 2$, respectively. The potential $C 1$ was generated semimicroscopically. The real part was calculated using a double-folding procedure by folding the projectile and target densities [21] with an effective $\mathrm{NN}$ interaction, whereas the imaginary part was parametrized in terms of a phenomenological Woods-Saxon potential. For the $\mathrm{Ni}$ target, the ${ }^{16} \mathrm{O}$ and ${ }^{58} \mathrm{Ni}$ densities were taken from Ref. [21], and for the NN interaction we adopted the M3Y parametrization [22]. For the imaginary part we used a volume Woods-Saxon potential with parameters adjusted in order to reproduce the elastic data of ${ }^{16} \mathrm{O}+{ }^{58} \mathrm{Ni}$ at $E=120 \mathrm{MeV}$ [23].

For the $\mathrm{Pb}$ target, the potential $C 1$ was also generated semimicroscopically, with the real part given by the São Paulo double-folding potential, using a ${ }^{16} \mathrm{O}$ density taken from electron scattering [24] and the ${ }^{208} \mathrm{~Pb}$ density from a Hartree-Fock calculation [25], whereas the imaginary part was also parametrized using a volume Woods-Saxon potential. The Woods-Saxon parameters as well as the normalization of the 
TABLE I. Optical model parameters for $p+{ }^{58} \mathrm{Ni}$ and $p+{ }^{208} \mathrm{~Pb}$ used in the CDCC calculations. The parameters $V_{0}, r_{0}$, and $a_{0}$ refer to the depth, the reduced radius, and the diffuseness of the real part, $W_{v}, r_{i}, a_{i}$ correspond to the volume imaginary part, and $W_{s}, r_{s}$, and $a_{s}$ correspond to the surface imaginary part. Reduced radii $\left(r_{x}\right)$ are converted to absolute radii $\left(R_{x}\right)$ as $R_{x}=r_{x} A_{t}^{1 / 3}$.

\begin{tabular}{|c|c|c|c|c|c|c|c|c|c|c|c|}
\hline System & Set & $\begin{array}{c}V_{0} \\
(\mathrm{MeV})\end{array}$ & $\begin{array}{c}r_{0} \\
(\mathrm{fm})\end{array}$ & $\begin{array}{c}a_{0} \\
(\mathrm{fm})\end{array}$ & $\begin{array}{c}W_{v} \\
(\mathrm{MeV})\end{array}$ & $\begin{array}{c}r_{i} \\
(\mathrm{fm})\end{array}$ & $\begin{array}{c}a_{i} \\
(\mathrm{fm})\end{array}$ & $\begin{array}{c}W_{s} \\
(\mathrm{MeV})\end{array}$ & $\begin{array}{c}r_{s} \\
(\mathrm{fm})\end{array}$ & $\begin{array}{c}a_{s} \\
(\mathrm{fm})\end{array}$ & Ref. \\
\hline$p+{ }^{208} \mathrm{~Pb}$ & $P 1$ & 59.1 & 1.244 & 0.646 & 0.52 & 1.244 & 0.646 & 8.41 & 1.246 & 0.615 & [19] \\
\hline \multirow[t]{2}{*}{$p+{ }^{58} \mathrm{Ni}$} & $P 1$ & 56.7 & 1.192 & 0.663 & 0.61 & 1.192 & 0.663 & 7.4 & 1.282 & 0.55 & [19] \\
\hline & $P 2$ & 54.5 & 1.17 & 0.75 & - & - & - & 9.71 & 1.32 & 0.534 & [20] \\
\hline
\end{tabular}

real part were adjusted to reproduce the elastic scattering of ${ }^{16} \mathrm{O}+{ }^{208} \mathrm{~Pb}$ at $E=170 \mathrm{MeV}[26]$.

The ${ }^{16} \mathrm{O}-{ }^{58} \mathrm{Ni}$ and ${ }^{16} \mathrm{O}-{ }^{208} \mathrm{~Pb}$ potentials denoted $\mathrm{C} 2$ use standard volume Woods-Saxon forms for both the real and imaginary parts. For ${ }^{16} \mathrm{O}-{ }^{58} \mathrm{Ni}$, we used the geometry of Keeley et al. [23], which was slightly modified, along with the depths, in order to reproduce the ${ }^{16} \mathrm{O}+{ }^{58} \mathrm{Ni}$ elastic scattering data at $E=120 \mathrm{MeV}$ [23]. To reduce the number of free parameters, we used the same radius for the real and imaginary parts $\left(r_{0}=r_{i}=1.06 \mathrm{fm}\right)$ and likewise for the diffuseness parameter $\left(a_{0}=a_{i}=0.65 \mathrm{fm}\right)$. For ${ }^{16} \mathrm{O}-{ }^{208} \mathrm{~Pb}$, we kept the same imaginary found in set $C 1$, and we adjusted the parameters of the real part to reproduce the data for this system at $170 \mathrm{MeV}$ [26].

The parameters for the core potentials $C 1$ and $C 2$ are listed in Table II. Depending on the chosen parametrization for the proton-target and ${ }^{16} \mathrm{O}+$ target systems we considered three different sets in our calculations: $C 1+P 1$ (set 1), $C 2+P 1$ (set 2), and $C 2+P 2$ (set 3$)$. For each choice of the proton-target and ${ }^{16} \mathrm{O}$-target interactions, diagonal and transition coupling potentials are generated by folding the sum of the proton-target and core-target interactions with the internal states of the ${ }^{17} \mathrm{~F}$ nucleus, according to Eq. (1). These coupling potentials are expanded in multipoles $(\lambda)$, from $\lambda=0$ to a maximum value $\lambda_{\max }$ (see, for example, Eq. (8) of Ref. [12]). Unless stated otherwise, the calculations presented below use $\lambda_{\max }=5$ for both the Coulomb and nuclear parts. The CDCC calculations were performed with the coupled-channels code FRESCO [27].

\section{COMPARISON WITH EXPERIMENTAL DATA}

The calculated differential elastic cross sections, relative to the Rutherford cross section, are shown in Fig. 1. The solid circles are the data from Ref. [16]. The ${ }^{58} \mathrm{Ni}$ data have been multiplied by the 1.2 factor, as suggested in Ref. [16]. The open circles in the bottom panel correspond to the data from a previous experiment [28]. The solid, dashed, and dot-dashed lines correspond, respectively, to the calculations with the sets 1,2 , and 3. For both targets, the agreement with the data is very good, with set 1 providing a somewhat better agreement in the case of the ${ }^{58} \mathrm{Ni}$ target.

We consider now the breakup observables. In order to illustrate the range of excitation energies involved in the process, we plot in Fig. 2 the breakup cross section as a function of the $p+{ }^{16} \mathrm{O}$ relative energy for both targets. Although the calculations included partial waves up to $\ell_{\max }=5$, we show only the partial waves $\ell \leqslant 3$ for clarity. We have verified that convergence of the breakup cross section requires a maximum excitation energy of about $15 \mathrm{MeV}$ for the ${ }^{58} \mathrm{Ni}$ target and about $10 \mathrm{MeV}$ for the ${ }^{208} \mathrm{~Pb}$ target. The plot corresponds to the parameter set $P 1-C 1$. The lower excitation energies required in the ${ }^{208} \mathrm{~Pb}$ case is due to the fact that Coulomb couplings are more important in this case. The peak in the $d_{3 / 2}$ wave is due to the $3 / 2^{+}$resonance.

We now compare the calculated breakup cross sections with the data. The CDCC method provides the breakup cross section with respect to the center of mass of the outgoing $p$ and ${ }^{16} \mathrm{O}$ fragments, whereas the data of Ref. [16] are referred to the laboratory scattering angle of the $p+{ }^{16} \mathrm{O}$ system. Consequently, the calculations were transformed to the laboratory frame using the appropriate kinematical transformation.

In Fig. 3 we compare the experimental data with the CDCC calculations. The solid, dashed, and dot-dashed lines correspond to the calculations with the potential sets 1, 2, and 3 , respectively. For the ${ }^{58} \mathrm{Ni}$ target, the calculations are in reasonable agreement with the data, predicting the presence of a maximum around $9^{\circ}$ and some oscillatory behavior below

TABLE II. Optical model parameters for ${ }^{16} \mathrm{O}+{ }^{58} \mathrm{Ni}$ and ${ }^{16} \mathrm{O}+{ }^{208} \mathrm{~Pb}$ used in the CDCC calculations. The parameters have the same meaning as in the case of the proton-target potentials. Reduced radii $\left(r_{x}\right)$ are converted to absolute radii $\left(R_{x}\right)$ as $R_{x}=r_{x}\left(A_{p}^{1 / 3}+A_{t}^{1 / 3}\right)$.

\begin{tabular}{lcccccrr}
\hline \hline System & Set & $N_{r}$ & $\begin{array}{c}V_{0} \\
(\mathrm{MeV})\end{array}$ & $\begin{array}{c}r_{0} \\
(\mathrm{fm})\end{array}$ & $\begin{array}{c}a_{0} \\
(\mathrm{fm})\end{array}$ & $\begin{array}{c}W_{v} \\
(\mathrm{MeV})\end{array}$ & $\begin{array}{c}r_{i} \\
(\mathrm{fm})\end{array}$ \\
\hline${ }^{16} \mathrm{O}+{ }^{208} \mathrm{~Pb}$ & $C 1$ & 1.63 & & & & 20.3 & 1.31 \\
$(\mathrm{fm})$
\end{tabular}



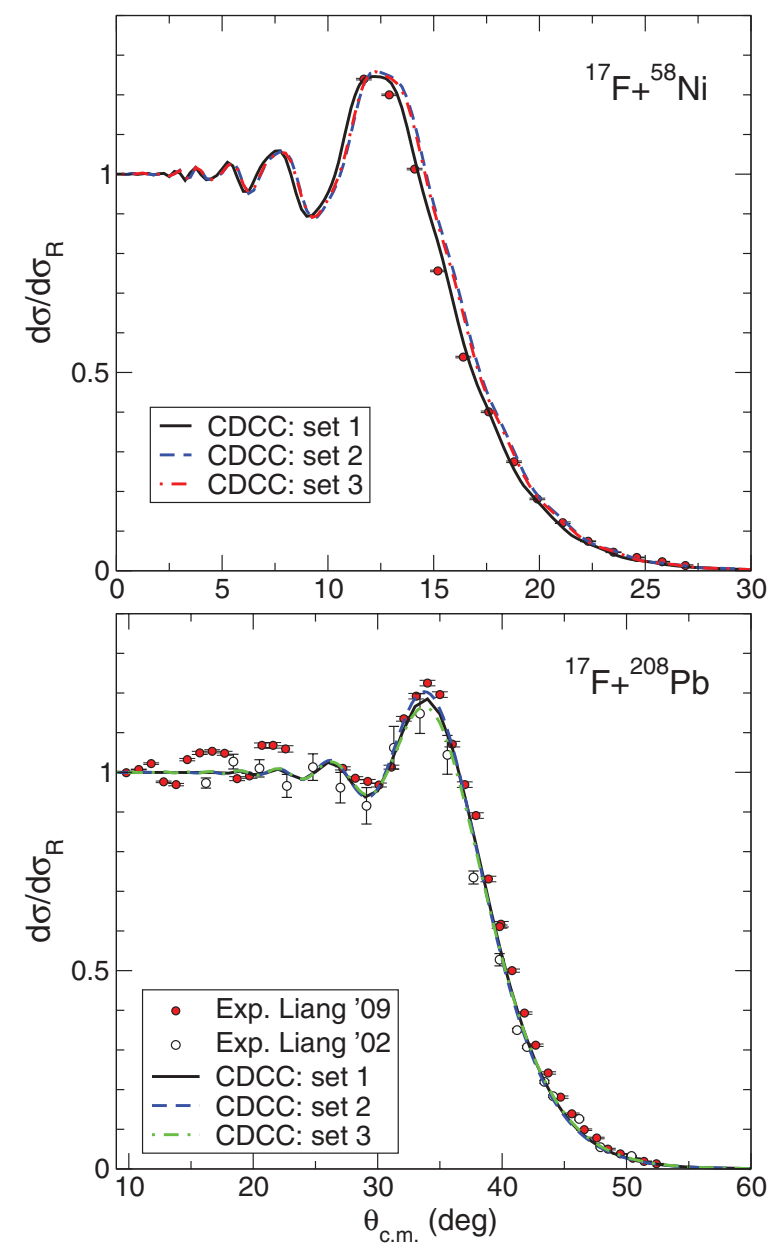

FIG. 1. (Color online) Differential elastic cross section, relative to Rutherford, for the scattering of ${ }^{17} \mathrm{~F}$ on ${ }^{58} \mathrm{Ni}$ (top panel) and ${ }^{208} \mathrm{~Pb}$ (bottom panel) at $10 \mathrm{MeV} /$ nucleon. The solid, dashed, and dot-dashed lines show the full CDCC calculations for sets 1, 2, and 3. The solid circles are the data from Ref. [16]. The open circles in the bottom panel are ${ }^{208} \mathrm{~Pb}$ data from a previous experiment [28].

this angle. This oscillatory structure cannot be confirmed with the present experiment due to the lack of data at very small angles. For angles beyond $10^{\circ}$, the calculations and the data display a smooth decrease, with the calculation slightly overestimating the data by a few percent. It is also observed that the three sets provide similar results, indicating a relatively weak sensitivity of the results with respect to the underlying fragment-target interactions. For set 1 the total breakup cross section is $21.5 \mathrm{mb}$, with differences of less than $10 \%$ for the two other sets. The calculated CDCC cross section agrees well with the dynamical calculation of Ref. [16] at the largest angles, but it is larger by a factor of almost 2 at the maximum. Moreover, the dynamical calculation displays a less oscillatory structure, as found in the comparison of Ref. [15].

For the ${ }^{208} \mathrm{~Pb}$ target, the calculations clearly overestimate the data. For example, the calculated differential cross section is about a factor of 2 larger than the data at the maximum. Sets 1 and 2 provide almost identical results. Set 3 yields a somewhat smaller cross section, which translates into slightly better agreement with the data. However, the magnitude is also
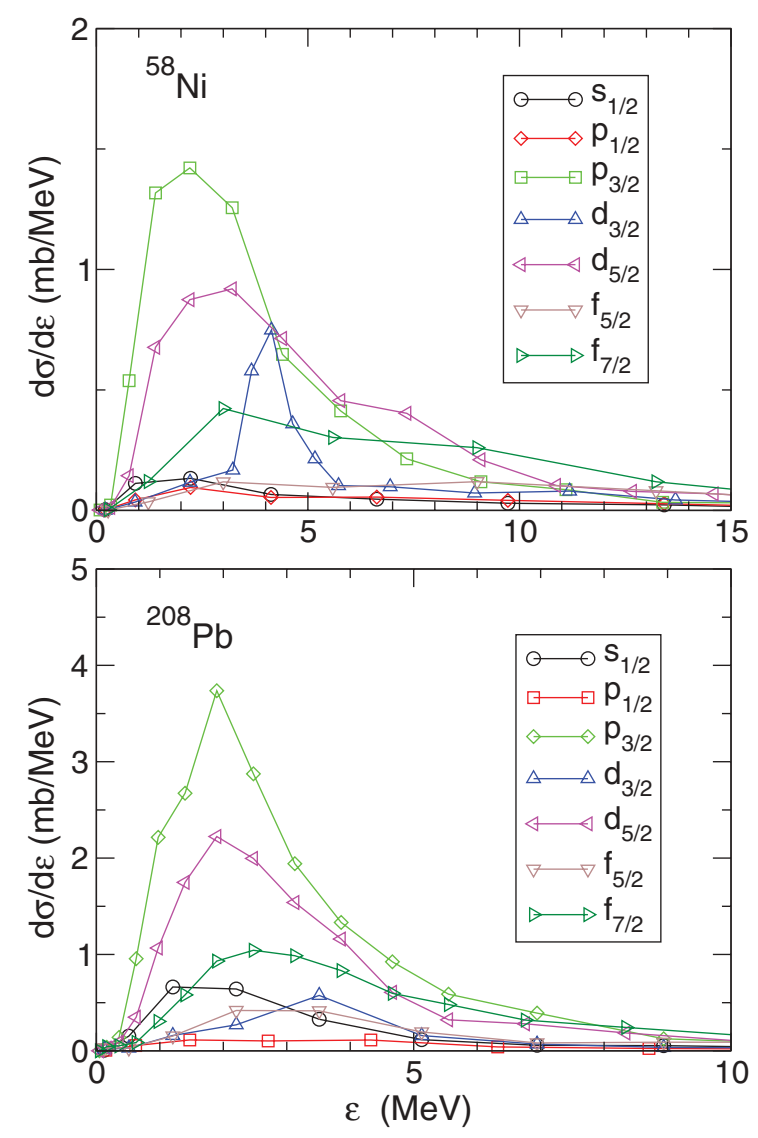

FIG. 2. (Color online) Breakup cross section as a function of the excitation energy (relative to the proton breakup threshold) for the ${ }^{58} \mathrm{Ni}$ (top) and ${ }^{208} \mathrm{~Pb}$ targets (bottom).

overestimated at intermediate angles. The angle-integrated cross sections are $\sim 32, \sim 31$, and $\sim 29 \mathrm{mb}$ for the sets 1,2 , and 3 , respectively. Interestingly, our CDCC results are very similar to the dynamical calculations shown in Ref. [16], in both shape and magnitude.

The disagreement between the calculations and the $\mathrm{Pb}$ data could be due to our simplified structure model for the ${ }^{17} \mathrm{~F}^{*}$ nucleus, in which the ${ }^{16} \mathrm{O}$ core is considered to be inert. In addition, part of the disagreement could be due to the uncertainties associated with the experimental extraction of the exclusive cross sections. This possibility is discussed further in the next section. Given the small differences found between the different parameter sets, all the calculations presented in the following are done with the 1 .

\section{FRAGMENT BREAKUP DISTRIBUTIONS}

In principle, the reconstruction of the exclusive cross section from the data would require the measurement of the protons and oxygen fragments in the full angular range or, at least, for all angles for which their respective cross sections are significant. However, due to the experimental arrangement used in the discussed experiment, the protons and oxygen fragments were recorded between the angles $8^{\circ}$ and $22^{\circ}$ for the ${ }^{58} \mathrm{Ni}$ target and $8^{\circ}$ and $50^{\circ}$ for the ${ }^{208} \mathrm{~Pb}$ target. This means that, in order to reconstruct the exclusive breakup spectrum 

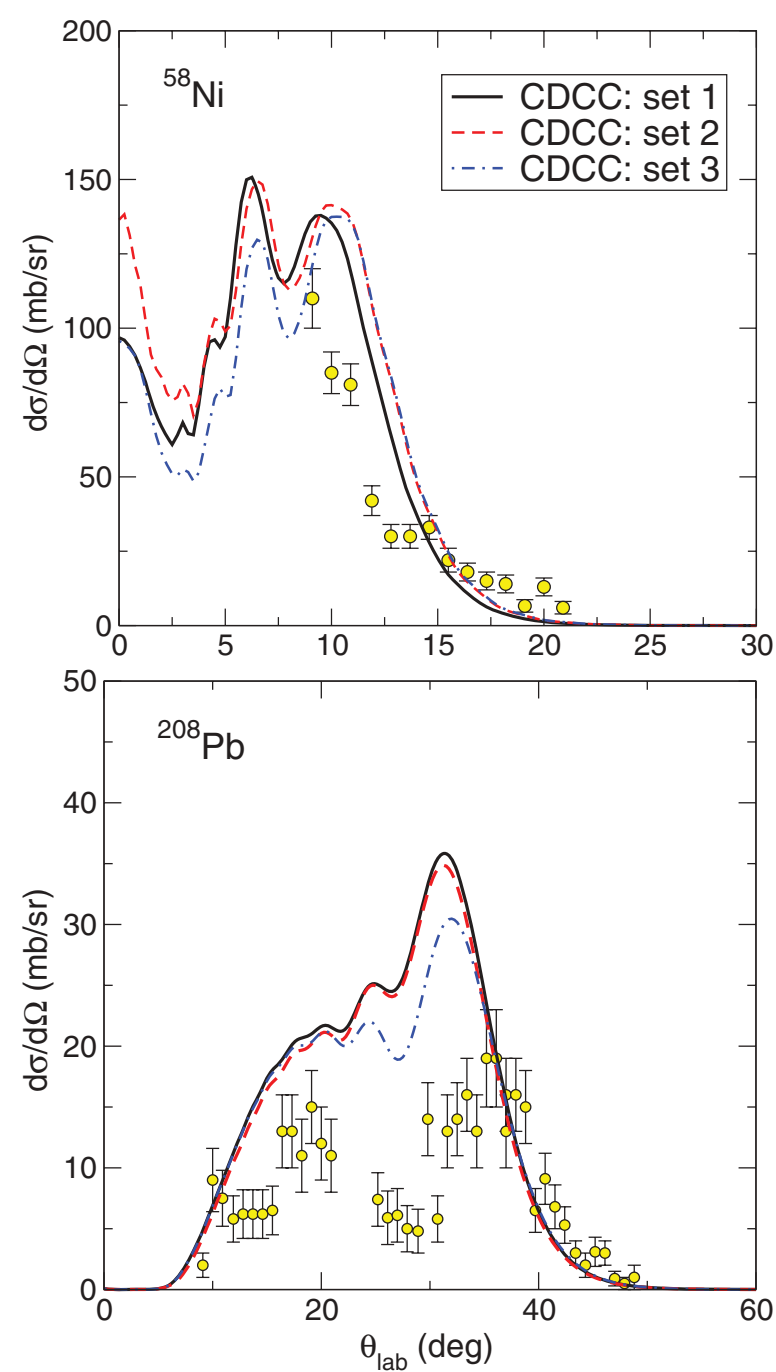

FIG. 3. (Color online) Differential breakup cross section as a function of the detection angle of the ${ }^{16} \mathrm{O}+p$ center of mass resulting from the scattering of ${ }^{17} \mathrm{~F}$ on ${ }^{58} \mathrm{Ni}$ (top) and ${ }^{208} \mathrm{~Pb}$ (bottom) at $10 \mathrm{MeV} /$ nucleon. The circles are the data from Ref. [16]. The solid, dashed, and dot-dashed lines are the CDCC calculations with the sets 1,2 , and 3 of parameters. See text for details.

with respect to the ${ }^{17} \mathrm{~F}^{*}$ scattering angle, one has to estimate in a reliable way the fraction of protons and oxygen fragments that are scattered outside the detector coverage. This requires an assumption about the angular distribution of the fragments. Although this estimation was carefully performed in the analysis of the studied experiment, this procedure necessarily brings some uncertainty to the extracted cross sections.

It is therefore illustrative to study the separate angular distribution for the protons and ${ }^{16} \mathrm{O}$ predicted by the present CDCC calculations. For this purpose we first calculate the triple differential cross section $d^{3} \sigma / d \Omega_{p} d \Omega_{c} d E_{c}$ where the subscript $p(c)$ refers to the proton (core). This is done using the formalism and codes developed in Ref. [29]. Then, this differential cross section is integrated in the energy of the core $\left(E_{c}\right)$ and in the angle of one of the fragments. This is shown in Fig. 4. The dotted and dashed lines correspond, respectively, to
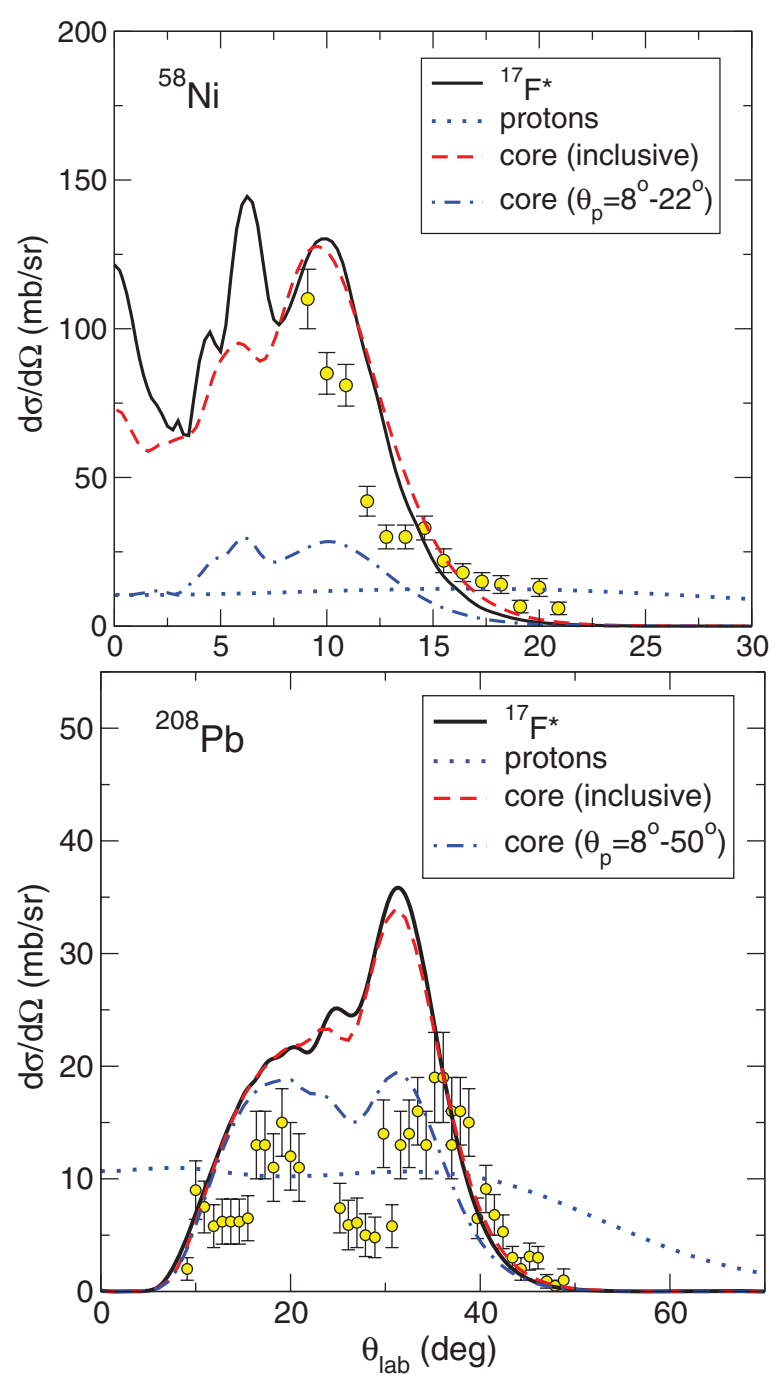

FIG. 4. (Color online) Proton and oxygen angular distribution. The solid line is the full CDCC calculation. The dotted and dashed lines are the inclusive proton and core angular distributions, respectively. The dot-dashed line is the core distribution in coincidence with protons between 8 and $22^{\circ}\left({ }^{58} \mathrm{Ni}\right)$ or 8 and $50^{\circ}\left({ }^{208} \mathrm{~Pb}\right)$. See text for details.

the proton and ${ }^{16} \mathrm{O}$ angular distributions. The dot-dashed line is the angular distribution for ${ }^{16} \mathrm{O}$ fragments in coincidence with protons scattered between angles $8^{\circ}$ and $22^{\circ}$ (for ${ }^{58} \mathrm{Ni}$ ) or $8^{\circ}$ and $50^{\circ}$ (for ${ }^{208} \mathrm{~Pb}$ ). One clearly sees that the inclusive ${ }^{16} \mathrm{O}$ distribution (integrated for all proton angles) follows very closely the ${ }^{17} \mathrm{~F}^{*}$ angular distribution and is concentrated within the angular range covered by the experiment. By contrast, the protons have a much broader angular distribution and a significant contribution is outside the experimental range. For the ${ }^{208} \mathrm{~Pb}$ target, the angle-integrated cross section, from $0^{\circ}$ to $180^{\circ}$, amounts to $\approx 38 \mathrm{mb}$, whereas for the $8^{\circ}-50^{\circ}$ range this is reduced to $\approx 22 \mathrm{mb}$. The estimation of the fraction of protons scattered outside the detection angles will add necessarily some uncertainty to the extracted exclusive cross section, which might also contribute to the disagreement with our calculations. 


\section{EFFECT OF DIFFERENT MULTIPOLES}

An interesting result of the present coupled-channels calculations is the appreciable contribution to the breakup cross section coming from high values of $\lambda$ in the multipole expansion of the coupling potentials given by Eq. (1). In fact, in order to achieve full convergence of the calculated observables, one has to include multipoles up to $\lambda \approx 5$. To illustrate this result, in Fig. 5 we compare the result of the CDCC calculation obtained with $\lambda_{\max }=5$ (thick solid line) with that obtained with $\lambda_{\max }=2$ (thin solid line). The difference between these two calculations indicates that there is still a non-negligible contribution from $\lambda>2$ multipoles.

We show also in this figure the CDCC calculations performed with a single multipole: $\lambda=0$ (dotted line), $\lambda=1$ (dashed line), and $\lambda=2$ (dot-dashed line). It is interesting to see that, for both targets, the contribution of these three multipoles is of similar magnitude. This result is particularly interesting for the ${ }^{208} \mathrm{~Pb}$ target, for which one might expect a dominance of the dipole couplings due to high charge of this nucleus. However, monopole as well as quadrupole couplings are of the same order of magnitude so the three multipoles are important insofar as the total breakup cross section is
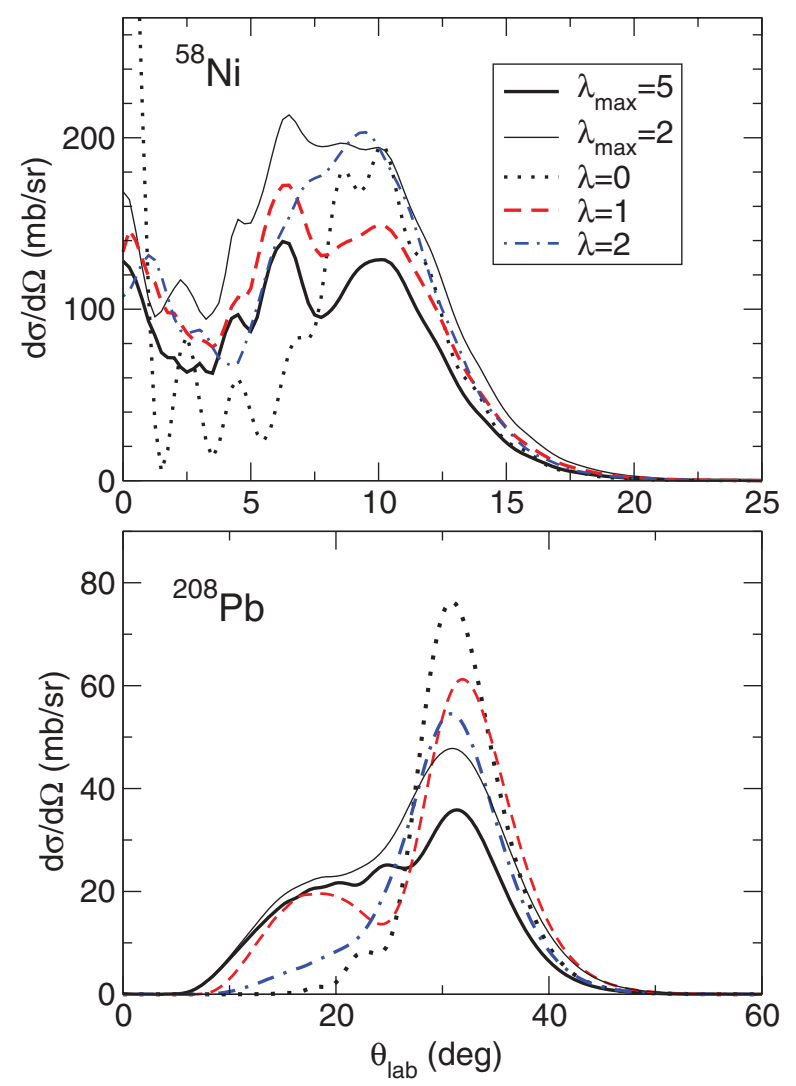

FIG. 5. (Color online) Contribution of the different coupling multipoles $(\lambda)$ to the exclusive breakup cross section. The thick and thin solid lines are the full CDCC calculations, including multipoles up to $\lambda_{\max }=5$ and $\lambda_{\max }=2$, respectively. The dotted, dashed, and dot-dashed lines are the calculations including only the $\lambda=0,1$, or 2 multipole, respectively. All these calculations include both nuclear and Coulomb couplings. concerned. Note that the large contribution arising from $\lambda=0$ comes from the nuclear couplings.

Comparing the calculations performed with a single multipole $(\lambda=0,1$, or 2$)$, with the $\lambda_{\max }=2$ calculation, it is also clear that there is a strong destructive interference between $\lambda=$ 0,1 , and 2 multipoles. This result casts doubt on the validity of the perturbative method to analyze these reactions, in which the contribution from different multipoles are added incoherently, as was done in the original analysis of these data [16].

The results presented in this section differ from those of Ref. [30], where a clear dominance of dipole couplings was found for the same reaction, but at a much higher energy (65 MeV/nucleon). A consequence of this result is that, for the purpose of extracting information on the corresponding capture reaction from the breakup with a heavy target, it is better to perform the experiment at higher incident energies, where the process is largely dominated by $E 1$ couplings.

\section{NUCLEAR VERSUS COULOMB BREAKUP}

The calculations presented in the preceding sections include both nuclear and Coulomb couplings. We have also studied the separate contribution of the nuclear and Coulomb interactions in the breakup cross section. The results are depicted in Fig. 6, where we compare the full CDCC calculation, including both nuclear and Coulomb couplings (solid line) with the CDCC calculations including only nuclear interactions (dashed line) or Coulomb interactions (dotted-dashed line). In the former, we keep also the monopole Coulomb potential, which does not lead to breakup but is important to preserve the center-of-mass motion of the projectile.

For both targets, the "only-Coulomb" calculation has a smooth angular dependence, extending to very large angles. In fact, this result would be similar to that obtained in the semiclassical theory of Coulomb excitation of Alder and Winther [5]. In the ${ }^{58} \mathrm{Ni}$ case, the "only-nuclear" calculation displays a very oscillatory pattern and grows at small angles. In addition, nuclear breakup is very small beyond the grazing angle $\left(\theta \approx 12^{\circ}\right)$ due to the absorption of the imaginary potentials. The coherent sum of Coulomb and nuclear couplings (solid line) shows also an oscillatory pattern. It is also apparent that the interference between the Coulomb and nuclear couplings is destructive at small angles and constructive around the maximum. This interference phenomenon between nuclear and Coulomb breakup has been reported in the past, for example, in the ${ }^{8} \mathrm{~B}+{ }^{58} \mathrm{Ni}$ reaction at $26 \mathrm{MeV}$ [12,31].

For the ${ }^{208} \mathrm{~Pb}$ target, the nuclear contribution has a bellshaped behavior, with a maximum around the grazing angle $\left(\theta \approx 35^{\circ}\right)$. Beyond this angle, the breakup probability drops quickly due to the absorption effects. The simultaneous inclusion of nuclear and Coulomb couplings produces some interference around $25^{\circ}$, where Coulomb and nuclear breakup are of similar magnitude. At small angles, the total breakup is clearly dominated by Coulomb couplings, whereas beyond the grazing angle, the Coulomb breakup is almost completely suppressed by the absorptive effects.

In the reactions studied in this work, the projectile-target Coulomb interaction arises from both the core and the valence proton. There have been several works trying to disentangle the 

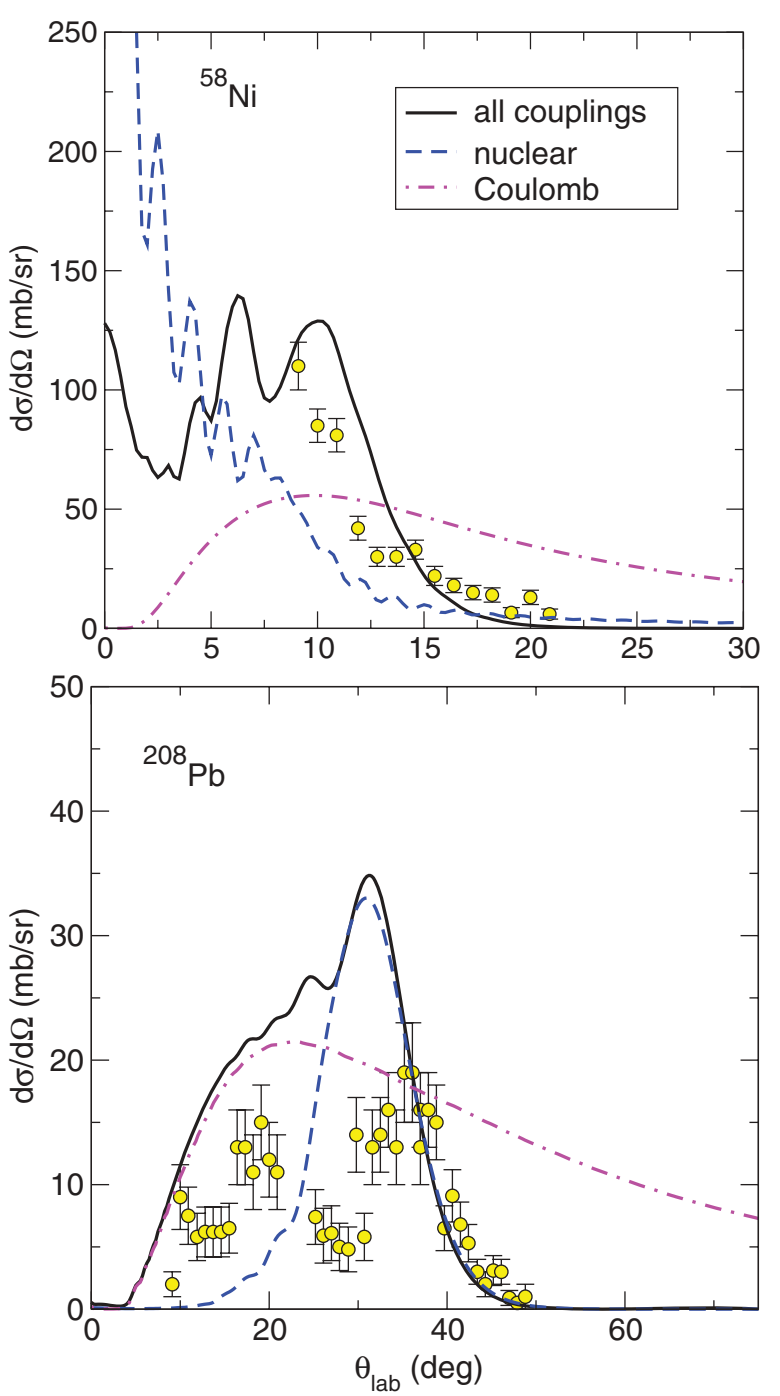

FIG. 6. (Color online) Nuclear (dashed) and Coulomb (dotdashed) contributions to the exclusive breakup cross section. The solid line is the full CDCC calculation, including both nuclear and Coulomb couplings.

separate role of the proton and core Coulomb potential in the scattering of proton-halo nuclei (e.g., [17,32,33]). Motivated by these works, we have performed a serial of calculations for different choices of the proton-target interaction. The results are depicted in Fig. 7 for the lead target, using a model space with $\ell \leqslant 3$ continuum waves. The thick solid line is the CDCC calculation performed with the full proton-target interaction, including both the nuclear (complex) and Coulomb parts. The dotted line is the CDCC calculation obtained by removing the proton-target interaction. Note that in this latter case, the breakup is entirely due to a core-recoil effect, that is, the displacement of the core with respect to the center of mass of the projectile. The angle-integrated breakup cross sections for these calculations are 33.5 and $41.6 \mathrm{mb}$, respectively.

One might naively expect that the inclusion of the protontarget interaction should increase the breakup cross section. To understand why this is not the case, we include in Fig. 7 three auxiliary CDCC calculations. First, we consider a calculation

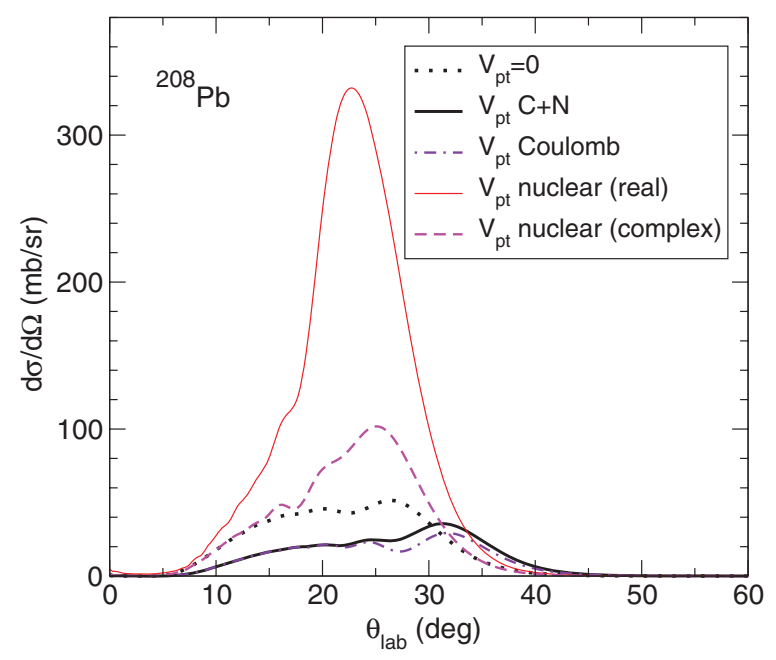

FIG. 7. (Color online) Breakup angular distribution for the ${ }^{17} \mathrm{~F}+$ ${ }^{208} \mathrm{~Pb}$ at $170 \mathrm{MeV}$ obtained in the CDCC calculation for different choices of the proton-target interaction, as specified by the labels.

retaining for the proton-target system only the real part of the nuclear interaction and without Coulomb potential (thin solid line). In this situation, the breakup cross section increases drastically ( $\sim 170 \mathrm{mb}$ ) with respect to the zero proton-target potential calculation. The effect is larger than the core recoil, owning to the larger displacement of the proton with respect to the projectile center of mass compared to the core. However, adding the imaginary part to the proton-target interaction (dashed line) reduces significantly the breakup cross section. This is because the imaginary part will produce an absorptive effect, which removes flux from the elastic and elastic-breakup channels in favor of other channels (such as nonelastic breakup). Finally, we consider a calculation in which we include the proton-target Coulomb potential but remove completely the nuclear part (dotted-dashed line). This produces also a hindrance of the breakup cross section, with respect to the zero potential. This phenomenon has been described by several authors $[16,32,33]$ and has been referred to as "shielding" or "dynamic polarization" effect. It has been interpreted as a result of the screening experienced by the proton when it is displaced behind the nuclear core, leading to a destructive interference between the proton and core Coulomb potentials [33].

We can summarize these results by saying that the effect of the real part of the proton-target interaction is to increase the elastic breakup cross section, whereas the imaginary part, as well as the Coulomb potential, produce a suppression of this breakup.

It has been suggested in Ref. [33] that a suitable observable to disentangle the Coulomb effects due to the proton-target and core-target interactions is the proton angular distribution after breakup, since interference effects between them were found to be small for this observable. It was also shown in that work that the core-recoil contribution to the proton angular distribution can be simulated by replacing the valence proton by a neutron with an effective separation energy. For the ${ }^{17} \mathrm{~F}+$ ${ }^{208} \mathrm{~Pb}$ reaction an effective binding energy of $1.7 \mathrm{MeV}$ was prescribed. Since the calculations of Ref. [33] correspond to a much higher energy (72 MeV/nucleon) we have investigated 


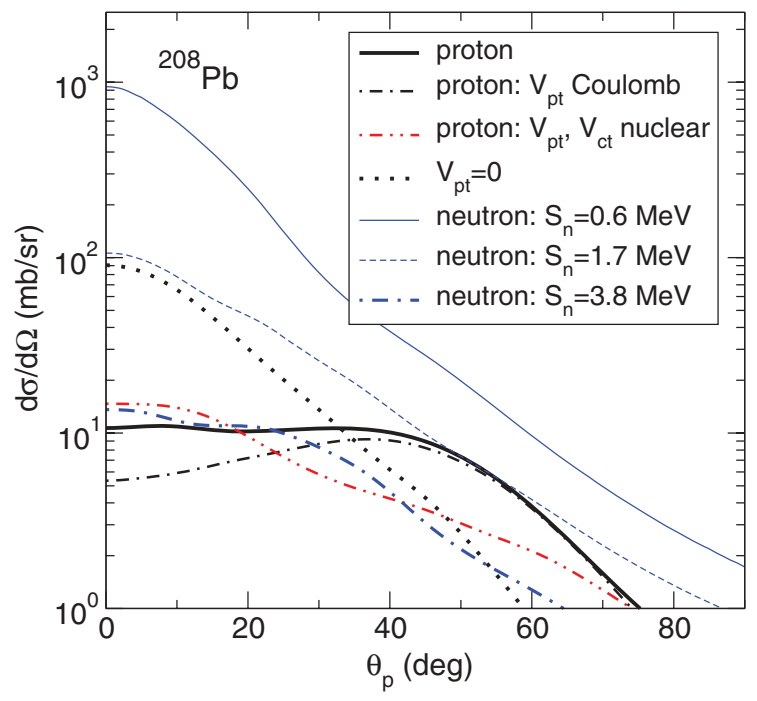

FIG. 8. (Color online) Proton angular distribution following the breakup of ${ }^{17} \mathrm{~F}$ on ${ }^{208} \mathrm{~Pb}$ at $170 \mathrm{MeV}$. See text for details.

the validity of these results at the energy of the present work ( $E_{\text {lab }}=170 \mathrm{MeV}$, that is, $\sim 10 \mathrm{MeV} /$ nucleon).

The results are shown in Fig. 8, where we present the proton angular distribution (in the laboratory frame) for different choices of the proton potential. In all these calculations, we consider only $\ell \leqslant 3$ continuum states. The thick solid line is the full CDCC calculation, including the nuclear and Coulomb parts of the proton-core and proton-target interactions. The thin dot-dashed line is the calculation removing the nuclear part (real and imaginary) of the proton-target potential. As shown in Fig. 7, this produces only a small decrease of the breakup cross section. If we now remove completely the proton-target potential (dotted line) the proton angular distribution departs significantly with respect to the full calculation, increasing at small angles by about one order of magnitude and decreasing at larger angles. This result is consistent with Fig. 7 and evidences once more the importance of the Coulomb part of the proton-target interaction. We present an additional proton calculation (double dot-dashed line) in which we retain only the nuclear parts of the proton-target and core-target interactions, that is, including only nuclear breakup. The remaining lines included in this figure (depicted with blue lines) are neutronlike calculations obtained by setting to zero the charge of the proton, that is, replacing the valence proton with a neutron. The thin solid line is the neutronlike calculation, keeping the experimental separation energy of the valence proton $(0.6 \mathrm{MeV})$. In this case, the breakup cross section increases significantly, particularly at small angles. The thick dashed line is the neutronlike calculation using an effective separation energy of $1.7 \mathrm{MeV}$. The thick dot-dashed line is the neutronlike calculation with the effective separation energy of $3.8 \mathrm{MeV}$, which was the value originally suggested in Ref. [17]. In this case, the neutron angular distribution is close to the full calculation at small angles and becomes closer to the core-recoil curve at larger angles. It becomes clear from these calculations that the shape of the proton angular distribution, in the full calculation, cannot be reproduced by any of the neutronlike calculations. This is because the presence of the
Coulomb part in the proton case produces interference effects with the core-target part which are not present in the neutron case. However, it can be seen that the neutronlike calculation with the effective separation energy of $3.8 \mathrm{MeV}$ is close to the proton calculation including only nuclear breakup (double dot-dashed line). From this result we conclude that, as far as the nuclear breakup is concerned, the proton dynamics is similar to that of a more bound neutron, in agreement with the results of Ref. [33].

We have also performed a similar comparison for the breakup angular distribution with respect to the center of mass of the outgoing ${ }^{17} \mathrm{~F}$ system. The results are presented in Fig. 9 for the lead target at two different incident energies: (a) $10 \mathrm{MeV} /$ nucleon and (b) $65 \mathrm{MeV} /$ nucleon. Since we are only interested in the comparison between different theoretical approaches, for the $65 \mathrm{MeV} /$ nucleon calculations we just use the same potentials used for the lower incident energy. The solid line represents the CDCC calculation for the actual ${ }^{17} \mathrm{~F}$ projectile, taking into account the Coulomb potential of the proton with the core and the target. The dashed and dot-dashed lines are the CDCC calculations setting to zero the charge of the valence proton and using effective binding energies of
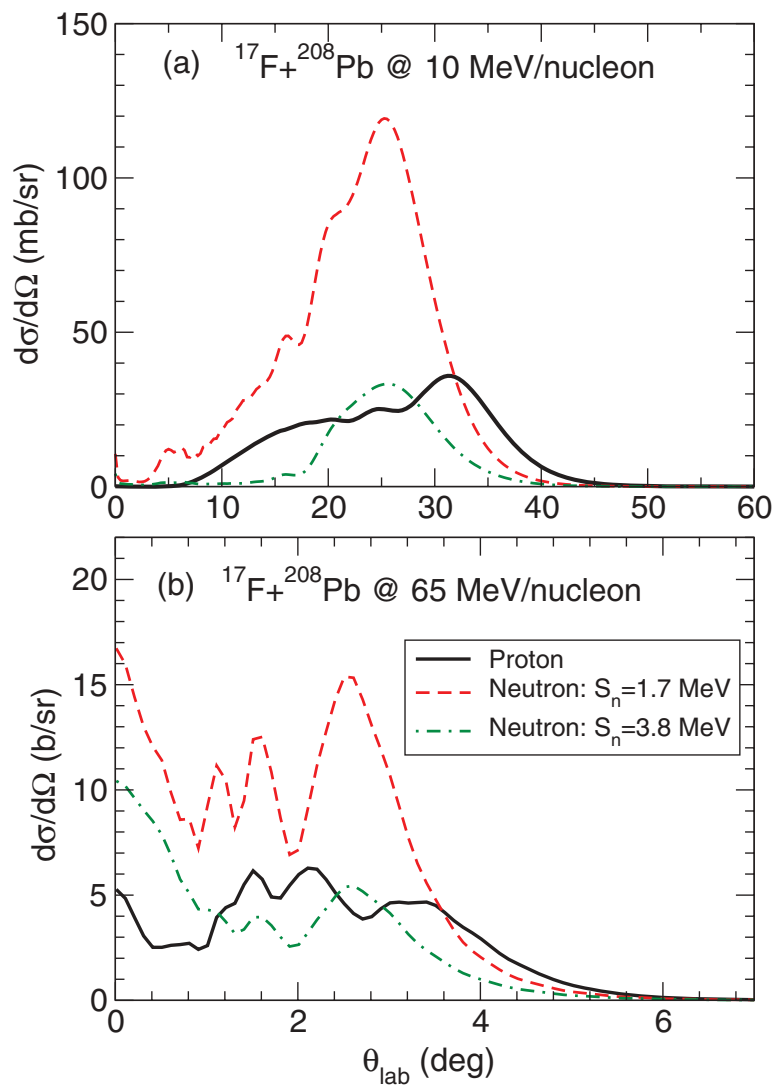

FIG. 9. (Color online) Angular distribution for the exclusive breakup of ${ }^{17} \mathrm{~F}+{ }^{208} \mathrm{~Pb}$ at (a) $10 \mathrm{MeV} /$ nucleon and (b) $65 \mathrm{MeV} /$ nucleon. Note the units in $\mathrm{b} / \mathrm{sr}$ for the latter. The solid line is the CDCC calculation for the actual ${ }^{17} \mathrm{~F}$ projectile, whereas the dashed and dot-dashed lines are the calculations obtained neglecting the charge of the valence proton and using an effective separation energy, as indicated by the labels. 
TABLE III. Total breakup cross sections (in mb) calculated for the ${ }^{17} \mathrm{~F}+{ }^{208} \mathrm{~Pb}$ reaction at $10 \mathrm{MeV} /$ nucleon and $65 \mathrm{MeV} /$ nucleon. Separation energies are given in $\mathrm{MeV}$.

\begin{tabular}{lcccc}
\hline \hline $\begin{array}{l}\text { Energy } \\
(\mathrm{MeV} / \text { nucleon })\end{array}$ & ${ }^{17} \mathrm{~F}$ & $\begin{array}{c}\text { Neutron } \\
\left(S_{n}=0.6\right)\end{array}$ & $\begin{array}{c}\text { Neutron } \\
\left(S_{n}=1.7\right)\end{array}$ & $\begin{array}{c}\text { Neutron } \\
\left(S_{n}=3.8\right)\end{array}$ \\
\hline 10 & 33 & 307 & 70 & 18 \\
65 & 86 & 334 & 149 & 59 \\
\hline \hline
\end{tabular}

1.7 $\mathrm{MeV}$ and 3.8 MeV, respectively. We provide also the total (angle and energy integrated) exclusive breakup cross sections obtained in these calculations in Table III. As expected, the calculation with $S_{n}=0.6 \mathrm{MeV}$ gives a much larger breakup cross section as compared to the proton case. Increasing its binding energy reduces the breakup cross section. The value obtained in the proton case is in between the values obtained in the neutron case for the two effective binding energies. However, we see in Fig. 9 that none of these values seems to reproduce well the angular distribution of the actual ${ }^{17} \mathrm{~F}$ case.

Thus, as in the case of the valence particle angular distribution, we can conclude that the projectile angular distribution is qualitatively different in the case of proton breakup compared to the neutron case. The fact that this difference remains, varying the binding energy for the neutron, indicates that its origin is not just a consequence of the different extensions of
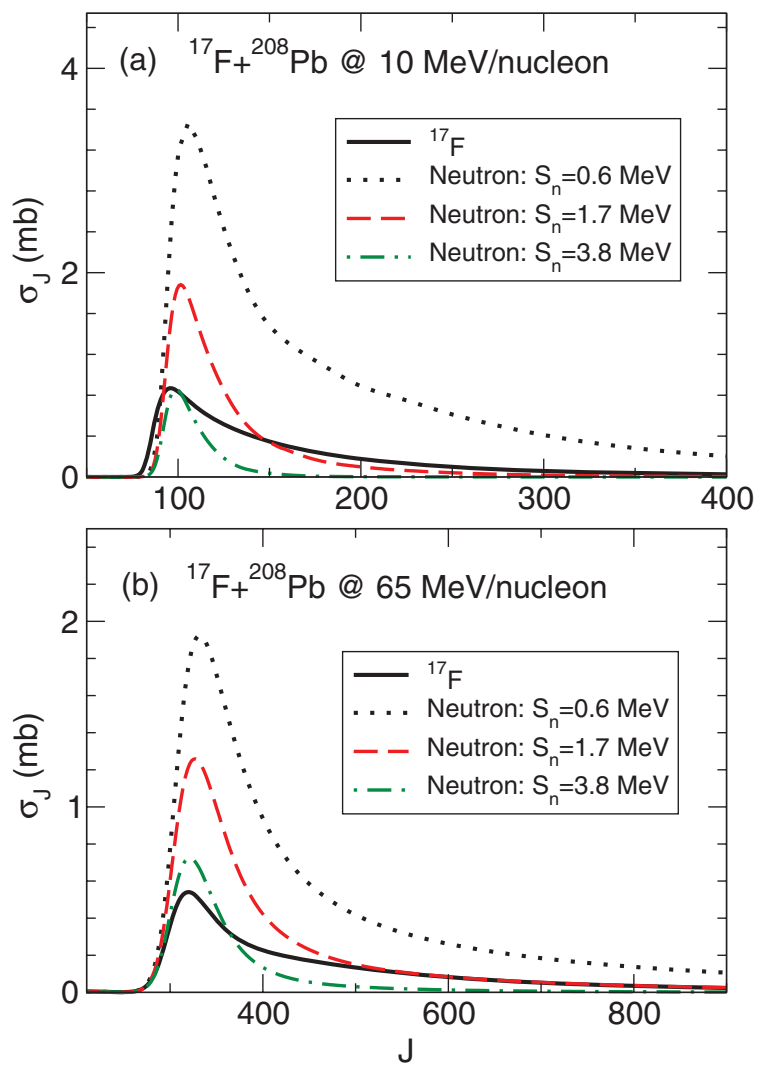

FIG. 10. (Color online) Angle and energy integrated breakup cross section, as a function of the total angular momentum $J$ for ${ }^{17} \mathrm{~F}+{ }^{208} \mathrm{~Pb}$ at (a) $10 \mathrm{MeV} /$ nucleon and (b) $65 \mathrm{MeV} /$ nucleon. The meaning of the lines is explained by the legends and in the text. the proton and neutron wave functions but that it has to do with the peculiar dynamical effects originated by the Coulomb part of the proton-target potential.

The get further insight into this result, we have studied also the total angular momentum $(J)$ dependence of the nonelastic cross section (inelastic + breakup). The results are shown in Fig. 10 for the two energies considered above. The neutronlike calculations with $S_{n}=0.6 \mathrm{MeV}$ overestimates by a large amount the proton cross section for all values of $J$. Increasing its separation energy to $1.7 \mathrm{MeV}$ improves the agreement with the proton case for the larger values of $J$ (large impact parameters), but this choice overestimates the cross section for values of $J$ around the maximum. Conversely, the choice 3.8 $\mathrm{MeV}$ reproduces well the cross section in this region but tends to underestimate the cross section for large $J$. It becomes clear that the discrepancy between the proton and neutron cases cannot be palliated with any choice of the binding energy in the neutronlike case. This explains the difference in shape of the corresponding angular distributions. Nevertheless, for reaction observables which are mainly sensitive to the large impact parameters, one may expect that the neutron model with the effective binding energy of about $1.7 \mathrm{MeV}$ can simulate the proton case results.

\section{SUMMARY AND CONCLUSIONS}

In this work, we have studied the scattering of the weakly bound nucleus ${ }^{17} \mathrm{~F}$ on ${ }^{58} \mathrm{Ni}$ and ${ }^{208} \mathrm{~Pb}$ targets at a incident energy of $10 \mathrm{MeV} /$ nucleon. Quasielastic and breakup data from a recent experiment [16] have been compared with CDCC calculations, assuming a two-body model for the ${ }^{17} \mathrm{~F}$ nucleus $\left({ }^{16} \mathrm{O}+p\right)$. For the ${ }^{58} \mathrm{Ni}$ target, the calculations reproduce very well the quasielastic data and they account also reasonably well for the exclusive breakup data. For the ${ }^{208} \mathrm{~Pb}$ target, the calculations reproduce also fairly well the quasielastic data, but they tend to overestimate the breakup data. Our results are consistent with those found in the semiclassical dynamical calculation performed in Ref. [16]. We have checked that the results are not very sensitive to the choice of the proton-target and ${ }^{16} \mathrm{O}$-target interactions, provided that these interactions are chosen to reproduce the corresponding elastic scattering at the same energy per nucleon.

We have discussed the angular distribution of the protons and oxygen fragments following the ${ }^{17} \mathrm{~F}$ dissociation. The ${ }^{16} \mathrm{O}$ distribution does not differ much from the ${ }^{17} \mathrm{~F}^{*}$ center of mass. On the other hand, the proton distribution displays a broad distribution, which goes well beyond the experimental coverage. This means that a reliable extraction of the exclusive ${ }^{17} \mathrm{~F}^{*}$ breakup cross section requires a good estimate of the protons which are scattered outside the detectors.

We have also analyzed the effect of the different multipoles in the coupling potentials. Although monopole, dipole, and quadrupole couplings provide the main contribution, higher multipoles $\left(\lambda_{\max } \approx 5\right)$ are required to get full convergence of the calculated breakup cross section. This makes it difficult to disentangle the structure and reaction effects from the measured data.

We have investigated the role of the Coulomb and nuclear couplings in the breakup cross sections. We find that both 
mechanisms contribute significantly to the breakup cross section and, moreover, the interference between them is very important. The roles of different parts of the proton-target interaction have been studied. We find that the Coulomb part of this interaction interferes destructively with the core-recoil contribution, producing a reduction of the breakup cross section, in agreement with previous findings [32,33]. The real and imaginary parts of the nuclear potential have been found to act in opposite directions; the real part produces a large increase of the elastic breakup cross section, but the inclusion of the imaginary part produces a suppression of this effect.

By switching off the charge of the valence proton, we have investigated the difference between the proton and neutron breakup. For the same binding energy, the breakup cross section is much larger in the neutron case. The effect can be partially attributed to the larger extension of the neutron wave function, but it is also related to the destructive interference of the proton-target and core-target Coulomb contributions. In fact, increasing the separation energy in the neutron case reduces the breakup cross section but does not reproduce well the shape of breakup angular distributions. These results indicate that the dynamics of the proton breakup is qualitatively different from that of the neutron case, in agreement with the conclusions of previous semiclassical calculations [33].

Finally, we speculate that a possible reason for the observed disagreement between our CDCC calculations and the exper- imental cross sections in the case of the lead target could be partially related to the treatment of the ${ }^{17} \mathrm{~F}$ states in a two-body model with an inert core. A natural improvement of this model would be the inclusion of core admixtures in the description of these states as well as the effect of core excitation in the reaction mechanism. The extension of the CDCC method to include this core excitation effects has been proposed and applied to some specific cases [34], but the complexity of these calculations has prevented so far their widespread use by the community. If would be interesting to apply these developments to the ${ }^{17} \mathrm{~F}+{ }^{208} \mathrm{~Pb}$ reaction to assess the importance of these effects.

\section{ACKNOWLEDGMENTS}

We are grateful to J. F. Liang for providing us the experimental data in tabular form and for support in their interpretation. We are also grateful to Aczel García Ríos for his help in the calculation of the double-folding calculations. Y. Kucuk acknowledges the financial support of the Turkish Science and Research Council (TÜBITAK) for postdoctoral research assistance through Grant No. BIDEB-2219 and also thanks I. Boztosun for computational help. This work was supported by TÜBITAK with Grant No. 110T388. A.M. is supported by the Spanish Ministerio de Ciencia e Innovación under Project No. FPA2009-07653.
[1] C. A. Bertulani and G. Baur, Nucl. Phys. A 442, 73 (1985).

[2] B. Davids et al., Phys. Rev. Lett. 86, 2750 (2001).

[3] F. Schumann et al., Phys. Rev. Lett. 90, 232501 (2003).

[4] T. Motobayashi et al., Phys. Rev. Lett. 73, 2680 (1994).

[5] K. Alder and A. Winther, Electromagnetic Excitation: The ory of Coulomb Excitation with Heavy Ions (North-Holland, Amsterdam, 1975).

[6] B. Davids, S. M. Austin, D. Bazin, H. Esbensen, B. M. Sherrill, I. J. Thompson, and J. A. Tostevin, Phys. Rev. C 63, 065806 (2001).

[7] T. Kido, K. Yabana, and Y. Suzuki, Phys. Rev. C 50, R1276 (1994).

[8] H. Esbensen, G. F. Bertsch, and C. A. Bertulani, Nucl. Phys. A 581, 107 (1995).

[9] H. Esbensen and G. F. Bertsch, Nucl. Phys. A 600, 37 (1996).

[10] A. Garcia-Camacho, G. Blanchon, A. Bonaccorso, and D. M. Brink, Phys. Rev. C 76, 014607 (2007).

[11] A. Garcia-Camacho, A. Bonaccorso, and D. M. Brink, Nucl. Phys. A 776, 118 (2006).

[12] F. M. Nunes and I. J. Thompson, Phys. Rev. C 59, 2652 (1999).

[13] J. Mortimer, I. J. Thompson, and J. A. Tostevin, Phys. Rev. C 65, 064619 (2002).

[14] N. Austern, Y. Iseri, M. Kamimura, M. Kawai, G. Rawitscher, and M. Yahiro, Phys. Rep. 154, 125 (1987).

[15] P. Capel, H. Esbensen, and F. M. Nunes, Phys. Rev. C 85, 044604 (2012).

[16] J. F. Liang, J. R. Beene, A. L. Caraley, H. Esbensen, A. GalindoUribarri, C. J. Gross, P. E. Mueller, K. T. Schmitt, D. Shapira, D. W. Stracener et al., Phys. Lett. B 681, 22 (2009).

[17] A. Bonaccorso, D. M. Brink, and C. A. Bertulani, Phys. Rev. C 69, 024615 (2004).
[18] J. M. Sparenberg, D. Baye, and B. Imanishi, Phys. Rev. C 61, 054610 (2000)

[19] A. J. Koning and J. P. Delaroche, Nucl. Phys. A 713, 231 (2003).

[20] F. D. Becchetti, Jr. and G. W. Greenless, Phys. Rev. 182, 1190 (1969).

[21] T. Belgya et al., Handbook for Calculations of Nuclear Reaction Data, RIPL-2 (IAEA, Vienna, 2006).

[22] G. Bertsch, J. Borysowicz, H. McManus, and W. Love, Nucl. Phys. A 284, 399 (1977).

[23] N. Keeley, J. A. Christley, N. M. Clarke, B. R. Fulton, J. S. Lilley, M. A. Nagarajan, and I. J. Thompson, Nucl. Phys. A 582, 314 (1995).

[24] C. W. De Jager, H. De Vries, and C. De Vries, At. Data Nucl. Data Tables 14, 479 (1974).

[25] J. W. Negele, Phys. Rev. C 1, 1260 (1969).

[26] S. D. Baker and J. A. McIntyre, Phys. Rev. 161, 1200 (1967).

[27] I. J. Thompson, Comp. Phys. Rep. 7, 167 (1988).

[28] J. F. Liang, J. R. Beene, H. Esbensen, A. Galindo-Uribarri, J. Gomez del Campo, C. J. Gross, M. L. Halbert, P. E. Mueller, D. Shapira, D. W. Stracener et al., Phys. Rev. C 65, 051603 (2002).

[29] J. A. Tostevin, F. M. Nunes, and I. J. Thompson, Phys. Rev. C 63, 024617 (2001).

[30] C. A. Bertulani and P. Danielewicz, Nucl. Phys. A 717, 199 (2003).

[31] F. M. Nunes and I. J. Thompson, Phys. Rev. C 57, R2818 (1998).

[32] H. Esbensen and G. F. Bertsch, Nucl. Phys. A 706, 383 (2002).

[33] R. Kumar and A. Bonaccorso, Phys. Rev. C 84, 014613 (2011).

[34] N. C. Summers, F. M. Nunes, and I. J. Thompson, Phys. Rev. C 74, 014606 (2006). 